\title{
Intracellular Theileria Parasites PIN Down Host Metabolism
}

\author{
Souhila Medjkane and Jonathan B. Weitzman *t \\ Université de Paris, UMR 7216 Epigenetics and Cell Fate, CNRS, Paris, France
}

Keywords: apicomplexa, parasite, Pin1, Theileria, PKM2, Fbw7, Warburg, metabolism

\section{INTRODUCTION}

The word "parasite" has been used since the 16th century, stemming from the Greek parasitos meaning "eating at another's table," from para- meaning "alongside" + sitos "food." The relationship between parasites and their hosts is an inherently metabolic one. Intracellular parasites feed off their host and are therefore likely to perturb their metabolic pathways in the process. Indeed, there has been much recent interest in the role of metabolic exchange in host-parasite interactions (Blume and Seeber, 2018; Zuzarte-Luís and Mota, 2018; Krishnan et al., 2019). In recent years we have become fascinated by a remarkable metabolic host-parasite interaction; Theileria parasites can reprogram their host cells to drive a cancer-like metabolic state. And our most exciting discovery

OPEN ACCESS

Edited by:

Jormay Lim,

National Taiwan University, Taiwan

Reviewed by:

Won Fen Wong,

University of Malaya, Malaysia

Futoshi Suizu,

Hokkaido University, Japan

*Correspondence:

Jonathan B. Weitzman jonathan.weitzman@u-paris.fr

tORCID:

Jonathan B. Weitzman orcid.org/0000-0001-8445-0102

Specialty section:

This article was submitted to Cell Growth and Division,

a section of the journal

Frontiers in Cell and Developmental

Biology

Received: 16 December 2019

Accepted: 17 February 2020

Published: 17 March 2020

Citation:

Medjkane S and Weitzman JB (2020) Intracellular Theileria Parasites PIN Down Host Metabolism.

Front. Cell Dev. Biol. 8:134. doi: 10.3389/fcell.2020.00134 has been the critical role that the Peptidyl-Prolyl Cis/Trans Isomerase Pin1 plays in this relationship.

\section{TALENTED THEILERIA PARASITES}

Theileria spp. are obligate, intracellular parasites belonging to the phylum of apicomplexa. Two Theileria species, T. parva, and T. annulata are bovine-specific pathogens that cause disease with considerable economic impact due to the high cost of treatment, the cost of anti-tick control, animal mortality, and decreased bovine production. Tropical Theileriosis kills over 1.1 million cattle per year and costs in the hundreds of millions of dollars. Infection by Theileria causes a lymphoproliferative disease in cows that has some clinical features of human leukemias (Tretina et al., 2015). T. annulata infects bovine B cells and macrophages, whereas the related species $T$. parva infects B and T lymphocytes. Theileria-infected cells are transformed and immortalized (Cheeseman and Weitzman, 2015; Tretina et al., 2015); they display cancer phenotypes such as uncontrolled proliferation, growth factor independence, and increased invasiveness and the ability to form metastases in immunodeficient mice (Tretina et al., 2015). Of particular interest, Theileria-dependent transformation is reversible; animals can be cured by treatment with the theilericidal drug Buparvaquone. Incubating Theileria-infected cells in vitro with Buparvaquone, diminishes the number of intracellular parasites in host leukocytes, which loose the transformed phenotypes, stop proliferating, and regain apoptosis sensitivity. To drive host cell transformation, the parasite manipulates the host cell signaling pathways that control cell proliferation and survival. Several signaling pathways were implicated, including c-Jun N-terminal Kinase (JNK) and host nuclear factors c-Myc, NF-kB, and AP-1 (Chaussepied et al., 1998; Heussler et al., 2002; Dessauge et al., 2005; Tretina et al., 2015). We showed that the Jun/AP-1 transcription factor maintains a critical oncogenic microRNA feedback loop (Marsolier et al., 2013). Another fascinating feature of Theileria-induced transformation is the induction of a metabolic signature characteristic of the "Warburg effect" observed in cancer cells (hereafter referred to as a Warburg-like effect) (Cairns et al., 2011; Medjkane and Weitzman, 2013; Medjkane et al., 2014; Metheni et al., 2015). The parasite-induced Warburg-like effect shows the classic signs of a shift from oxidative phosphorylation to aerobic glycolysis. We and others previously reported the central role of the Hypoxia-inducible factor 1 alpha $(\mathrm{HIF} 1 \alpha)$ in driving the expression of glycolytic enzymes and 
metabolic genes in infected cells (Medjkane et al., 2014; Metheni et al., 2015). Despite this progress in identifying host pathways underlying the transformed phenotype, it remained unclear how the intracellular parasite initiates the signaling events leadings to rewiring of the host transcriptome.

\section{UNDERPINNING HOST-PARASITE INTERACTION}

In order to identify potential secreted oncoproteins in the $T$. annulata, we mined the parasite genome looking for genes encoding proteins with signal peptides that might be secreted into the host cytoplasm and acts as "epigenators" (Cheeseman and Weitzman, 2015) of oncogenic signals to hijack host regulatory pathways. A bioinformatics pipeline led to a relatively restricted list of candidate genes and the most promising on the list was the parasite homolog of Phosphorylation-Dependent PeptidylProlyl Cis/Trans Isomerase PIN1 (Marsolier et al., 2015). The role of human Pin 1 in carcinogenesis and metabolic reprogramming offered a link between infection and transformation by Theileria parasites (Nakatsu et al., 2019). The parasite encoded isomerase, that we named TaPin 1, is particularly interesting; it has a catalytic isomerase domain and the WW domain present in mammalian Pin 1 is replaced by a putative signal peptide sequence. While several Pin1 homologs also lack the WW domain, the PPIase domain of TaPin 1 is well conserved. Indeed, the TaPin1 PPIase domain shares $47 \%$ identity with hPin 1, 45\% with Arabidopsis thaliana AtPin1, and 43\% with Trypanosoma brucei TbPin1 (Marsolier et al., 2015). Interestingly, the signal peptide is not conserved in non-transforming species of Theileria or in the related apicomplexan homologs in Toxoplasma or Plasmodium (Marsolier et al., 2015). We showed that the TaPin 1 protein is a bona fide prolyl isomerase and that it is secreted into host cells (Marsolier et al., 2015). The importance of TaPin 1 in the parasite-induced transformation process was highlighted by the discovery that TaPin 1 isomerase activity can be inhibited by the anti-parasite drug Buparvaquone. An additional twist was the finding that Buparvaquone-resistant parasites have a mutation in the gene encoding TaPin 1 . The same A53P mutation has now been reported in drug-resistant isolates from both Tunisia and Sudan (Marsolier et al., 2015; Salim et al., 2019). This mutation affects the ability of Buparvaquone to enter into the active site and inhibit isomerase activity. Interestingly, the presence of a signal peptide was observed only in the transforming species ( $T$. annulata and T. parva), but not in non-transforming species or closely related apicomplexan such as Plasmodium or Toxoplasma (Marsolier et al., 2015). Although there are likely to be other parasite-encoded proteins that contribute to the transformation of the host cells, TaPin1 represents a remarkable of example of how a prolyl isomerase has evolved to play a key role in host-parasite relationships.

\section{TaPin1, A MOLECULAR LYNCHPIN}

Once TaPin1 was identified as a critical parasite-secreted epigenator, the question remained how it could hijack host cell

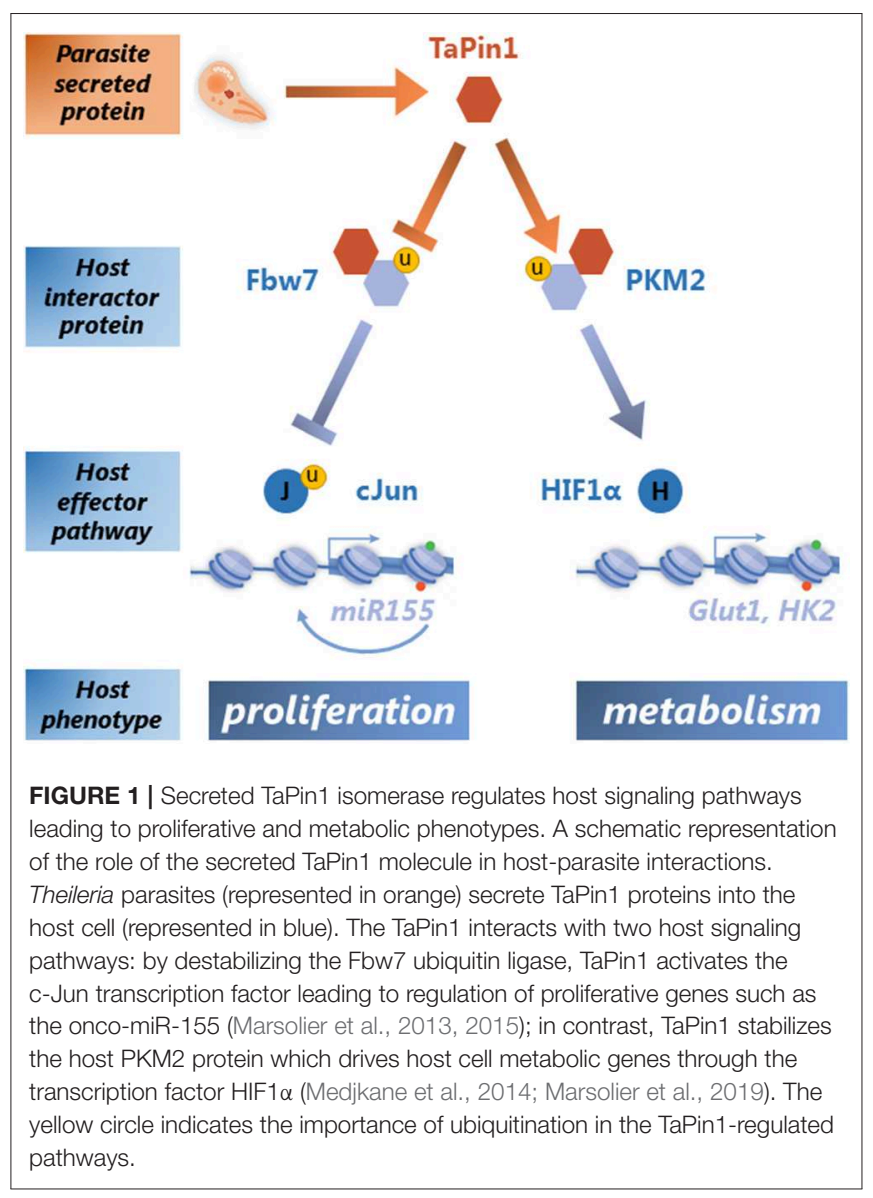

signaling pathways. Pin1 is a conserved enzyme that specifically isomerizes phosphorylated Ser/Thr-Pro bonds in a defined subset of proteins, thereby inducing conformational changes impacting their stability, localization and activity. Human Pin1 protein has multiple substrates involved in a wide range of cellular processes that contribute to transformation (Marsolier and Weitzman, 2014; Zhou and Lu, 2016). A search for TaPin1 interactors and host partner proteins identified at least two host pathways that are induced by the parasite isomerase (Figure 1). We showed that the TaPin1 protein interacts with host ubiquitin ligase Fbw7, leading to its auto-degradation (Marsolier et al., 2015). This interaction releases the host oncoprotein c-Jun from Fbw7-dependent ubiquitination and degradation. The c-Jun protein is part of the AP-1 transcription factor that induces the oncomiR-155 which drives host cell proliferation (Marsolier et al., 2013). AP-1 also induces the gene encoding the matrix metalloprotease MMP-9 which drives host cell invasive phenotypes (Cock-Rada et al., 2012). We also identified the host protein Pyruvate Kinase isoform M2 (PKM2), which is critical for the Warburg-like effect and the transcription of glycolytic enzymes in cancer cells, as a TaPin1 interactor (Marsolier et al., 2019). This time the consequence is the stabilization of PKM2 which leads to HIF- $1 \alpha$-dependent regulation of host metabolism. The TaPin1-PKM2-HIF-1 $\alpha$ axis causes induction of host metabolic enzymes (such as GLUT1 and Hexokinase 2), 
increased glucose uptake and the transformed phenotypes of parasite-infected cells (Medjkane et al., 2014; Marsolier et al., 2019). These are the combined features of the parasite-induced Warburg-like effect. The precise molecular mechanisms by which TaPin1 stabilizes host PKM2 protein, while promoting Fbw7 degradation, is unclear. We hypothesize that the prolyl isomerisation of PKM2 or Fbw7 could differentially affect the interaction with ubiquitin ligases or other factors that modulate protein stability.

\section{DISCUSSION}

Many studies on the role of the Pin 1 phosphorylation-dependent Peptidyl-Prolyl Cis/Trans Isomerase have firmly placed the protein as a key regulator of oncogenic and metabolic pathways (Marsolier and Weitzman, 2014; Zhou and Lu, 2016; Nakatsu et al., 2019). The discovery and characterization of the parasite TaPin1 add parasite-host interactions to the list of effects of this multi-tasking enzyme. As described above, TaPin1 links parasitism to the regulation of host metabolism and host cell proliferation. Our findings on TaPin 1 binding and isomerization of host substrates converge on the regulation of strategic host transcriptional reprogramming leading to two major biological processes that offer clear advantages for the parasite (Figure 1). First, TaPin 1 contributes to host cell proliferation and tumor growth via stabilization of c-Jun which promotes transformation, thereby enabling parasite dissemination. Secondly, TaPin1 induces major metabolic reprogramming through activation of the PKM2-HIF1 $\alpha$ axis. This shift in cellular glucose resources could potentially provide critical nutrients required for Theileria proliferation and maintenance within the host cells. Interestingly, the acquisition during evolution of a signal peptide for TaPin 1 that is restricted to transforming Theileria species ( $T$. annulata and $T$. parva) provides a compelling way to be secreted into the cytoplasmic host compartment in order to hijack transduction

\section{REFERENCES}

Blume, M., and Seeber, F. (2018). Metabolic interactions between Toxoplasma gondii and its host. F1000Res 7:F1000 Faculty Rev-1719. doi: 10.12688/f1000research.16021.1

Cairns, R. A., Harris, I. S., and Mak, T. W. (2011). Regulation of cancer cell metabolism. Nat. Rev. Cancer 11, 85-95. doi: 10.1038/nrc2981

Chaussepied, M., Lallemand, D., Moreau, M. F., Adamson, R., Hall, R., and Langsley, G. (1998). Upregulation of Jun and Fos family members and permanent JNK activity lead to constitutive AP-1 activation in Theileria-transformed leukocytes. Mol. Biochem. Parasitol. 94, 215-26. doi: 10.1016/S0166-6851(98)00070-X

Cheeseman, K., and Weitzman, J. B. (2015). Host-parasite interactions: an intimate epigenetic relationship. Cell. Microbiol. 17, 1121-1132. doi: 10.1111/cmi.12471

Cock-Rada, A. M., Medjkane, S., Janski, N., Yousfi, N., Perichon, M., Chaussepied, M., et al. (2012). SMYD3 promotes cancer invasion by epigenetic upregulation of the metalloproteinase MMP-9. Cancer Res. 72, 810-820. doi: 10.1158/0008-5472.CAN-11-1052

Dessauge, F., Hilaly, S., Baumgartner, M., Blumen, B., Werling, D., and Langsley, G. (2005). c-Myc activation by Theileria parasites promotes survival of infected B-lymphocytes. Oncogene 24, 1075-1083. doi: 10.1038/sj.onc. 1208314 pathways and rewire host transcriptional programs. In this way TaPin 1 is critical for parasite survival and is a promising drug target. Indeed, the observation in the field of Buparvaquoneresistant parasites and mutations in the TaPin 1 gene highlights the need for alternative Pin1 inhibitors that can still target mutant proteins. The levels of host bovine BtPin1 transcripts and protein were unaffected by Buparvaquone treatment, suggesting that this drug specifically targets the parasite protein and this might explain the absence of toxicity in uninfected cells. Of note, Juglone, a well-characterized inhibitor of mammalian Pin1 can substitute for the treatment by Buparvaquone leading to a decrease in parasite burden and viability of host cells infected with T. annulata or T. parva in vitro (Marsolier et al., 2015). Clearly, Pin1 proteins from different species will continue to amaze us with their versatility and multi-tasking in the years ahead. This is likely to remain an exciting field, with clinical relevance for both cancer and infectious diseases.

\section{AUTHOR CONTRIBUTIONS}

SM and JW wrote the article.

\section{FUNDING}

Work in our laboratory was supported by the LabEx Who Am I? \#ANR-11-LABX-0071 and the Université de Paris IdEx \#ANR18-IDEX-0001 funded by the French Government through its Investments for the Future program, the Agence Nationale de la Recherche (ANR PATHO-METHYLOME \#ANR-15-CE120020), the Plan Cancer Epigénétique et cancer 2015 (PARACAN \#PARA-15-RCA) the Fondation ARC pour la Recherche sur le Cancer (ARC $\mathrm{n}^{\circ} 155029$ ), and Gefluc les entreprises contre le cancer. JW is a Senior Member of the Institut Universitaire de France (IUF) and SM was a Junior member of the IUF (2012ND 3369).
Heussler, V. T., Rottenberg, S., Schwab, R., Küenzi, P., Fernandez, P. C., McKellar, S., et al. (2002). Hijacking of host cell IKK signalosomes by the transforming parasite Theileria. Science 298, 1033-1036. doi: 10.1126/science.1075462

Krishnan, A., Kloehn, J., Lunghi, M., and Soldati-Favre, D. (2019). Vitamin and cofactor acquisition in apicomplexans: synthesis versus salvage. J. Biol. Chem. 295, 701-714. doi: 10.1074/jbc.AW119.008150

Marsolier, J., Perichon, M., DeBarry, J. D., Villoutreix, B. O., Chluba, J., Lopez, T., et al. (2015). Theileria parasites secrete a prolyl isomerase to maintain host leukocyte transformation. Nature 520, 378-82. doi: 10.1038/nature 14044

Marsolier, J., Perichon, M., Weitzman, J. B., and Medjkane, S. (2019). Secreted parasite Pin1 isomerase stabilizes host PKM2 to reprogram host cell metabolism. Commun. Biol. 2:152. doi: 10.1038/s42003-019-0386-6

Marsolier, J., Pineau, S., Medjkane, S., Perichon, M., Yin, Q., Flemington, E., et al. (2013). OncomiR addiction is generated by a miR-155 feedback loop in theileria-transformed leukocytes. PLoS Pathog. 9:e1003222. doi: 10.1371/journal.ppat.1003222

Marsolier, J., and Weitzman, J. B. (2014). Pin1: a multi-talented peptidyl prolyl cistrans isomerase and a promising therapeutic target for human cancers. Med. Sci. 30, 772-778. doi: 10.1051/medsci/20143008015

Medjkane, S., Perichon, M., Marsolier, J., Dairou, J., and Weitzman, J. B. (2014). Theileria induces oxidative stress and HIF1 $\alpha$ activation that 
are essential for host leukocyte transformation. Oncogene 33, 1809-1817. doi: 10.1038/onc.2013.134

Medjkane, S., and Weitzman, J. B. (2013). A reversible Warburg effect is induced by Theileria parasites to transform host leukocytes. Cell Cycle 12, 2167-2168. doi: $10.4161 /$ cc. 25540

Metheni, M., Lombès, A., Bouillaud, F., Batteux, F., and Langsley, G. (2015). HIF$1 \alpha$ induction, proliferation and glycolysis of Theileria-infected leukocytes. Cell. Microbiol. 17, 467-472. doi: 10.1111/cmi.12421

Nakatsu, Y., Yamamotoya, T., Ueda, K., Ono, H., Inoue, M.-K., Matsunaga, Y., et al. (2019). Prolyl isomerase Pin1 in metabolic reprogramming of cancer cells. Cancer Lett. 420, 106-114. doi: 10.1016/j.canlet.2019.10.043

Salim, B., Chatanga, E., Jannot, G., Mossaad, E., Nakao, R., and Weitzman, J. B. (2019). Mutations in the TaPIN1 peptidyl prolyl isomerase gene in Theileria annulata parasites isolated in Sudan. Int. J. Parasitol. Drugs Drug Resist. 11, 101-105. doi: 10.1016/j.ijpddr.2019. 11.001

Tretina, K., Gotia, H. T., Mann, D. J., and Silva, J. C. (2015). Theileria-transformed bovine leukocytes have cancer hallmarks. Trends Parasitol. 31, 306-314. doi: 10.1016/j.pt.2015.04.001
Zhou, X. Z., and Lu, K. P. (2016). The isomerase PIN1 controls numerous cancer-driving pathways and is a unique drug target. Nat. Rev. Cancer 16, 463-478. doi: 10.1038/nrc. 2016.49

Zuzarte-Luís, V., and Mota, M. M. (2018). Parasite sensing of host nutrients and environmental cues. Cell Host Microbe 23, 749-758. doi: 10.1016/j.chom.2018.05.018

Conflict of Interest: The authors declare that the research was conducted in the absence of any commercial or financial relationships that could be construed as a potential conflict of interest.

Copyright (c) 2020 Medjkane and Weitzman. This is an open-access article distributed under the terms of the Creative Commons Attribution License (CC BY). The use, distribution or reproduction in other forums is permitted, provided the original author(s) and the copyright owner(s) are credited and that the original publication in this journal is cited, in accordance with accepted academic practice. No use, distribution or reproduction is permitted which does not comply with these terms. 